\title{
Chasing light from the crest of a wave
}

\author{
The Gravitational-wave Optical Transient Observer telescope will provide a rapid response to gravitational \\ wave event triggers in order to locate optical counterparts for subsequent multi-wavelength follow-up, \\ explains Danny Steeghs.
}

T he past few years have seen the longawaited era of gravitational wave (GW) astrophysics not just kick off, but proceed at a remarkable pace, with the Laser Interferometer Gravitational-Wave Observatory (LIGO) and Virgo consortium announcing several firm detections arising from black hole-black hole mergers. Corresponding signals at electromagnetic (EM) wavelengths were also expected for merger events involving neutron stars, promising a unique combination of constraints using both GW and EM measurements. We have just seen this hope vindicated by event GW 170817 (B. P. Abbott et al. Phys. Rev. Lett. 119, 161101; 2017). This event was not only the first binary neutron star merger detected in GWs, but also offered a rich variety of EM signals in the days following the GW signal (B. P. Abbott et al. Astrophys. J. Lett. 848, L12; 2017).

The ability of advanced gravitational wave detectors such as LIGO and Virgo to constrain the direction of the sources responsible for gravitational waves is limited. Thus, significant areas of the sky need to be scanned and searched over promptly in order to identify counterparts consistent with the GW signal. It is this specific challenge that was the motivation behind the Gravitationalwave Optical Transient Observer (GOTO) and drove the design decisions behind it. The sooner a viable EM counterpart can be identified following a GW detection, the more opportunities we have to study the inevitably short-lived source and its environment with our armada of follow-up telescopes and satellites. It was indeed thanks to a relatively good localization for GW 170817, that this challenge was made a little easier. For many events the expected search areas will be much larger.

GOTO employs an array of relatively modest wide-field optical telescopes with apertures of $40 \mathrm{~cm}$ and fast focal ratios whose individual fields of view $\left(5 \mathrm{deg}^{2}\right)$ can be combined to build up sky coverage. Its goal is to sweep large areas on the sky multiple times per night to a depth that should enable it to detect optical components from mergers within $100 \mathrm{Mpc}$ or so - the horizon distances that are expected to be achieved by the GW arrays. A custom equatorial robotic mount has been developed that can hold up to eight such telescopes within a single dome enclosure, saving costs and avoiding the complexity of needing to operate many individual mounts within their own enclosures. This concept means the project is scalable: more unit telescopes can be deployed to build up capability, and different trade-offs can be made between field of view, survey speed, depth or filter coverage across the combined array. This is not only cost effective, but also ensures that the operational mode can evolve as we learn more about the properties of the EM signals.

The first components of the GOTO project (Fig. 1) were installed in June 2017 and are currently being commissioned at the Roque de los Muchachos Observatory on the island of La Palma, Spain. Survey work is expected to commence in late 2017. The facility is linked to a data centre at the University of Warwick in the UK by a fast network link. In the present initial phases, four telescopes are mounted on a shared mount, offering a field of view of up to $20 \mathrm{deg}^{2}$. An additional four telescopes that can be accommodated on the same mount are expected in early 2018. More are planned: a fully deployed site incorporates 16 telescopes split over two mounts and domes; an additional site in the Southern Hemisphere would be desirable to ensure full coverage of the sky.

The push towards even more telescopes addresses a second challenge in this effort: dealing with false positives. In order to identify viable counterparts, one needs to search a sizeable proportion of the sky. But the sky is full of variable and transient phenomena, and thus we need to be wary of unrelated sources that are active in the target area close in time to the GW detection. GOTO will spend most of its time in patrol mode in order to provide us with crucial information about ongoing activity in the sky in anticipation of GW signals. This in itself will be an excellent data set offering insights into a variety of shortlived phenomena, but will also increase the reliability and speed with which we can expect to identify viable GW counterparts. Faster is indeed better in this case. Over the next few months, the GOTO team will work hard to optimize its observing and data

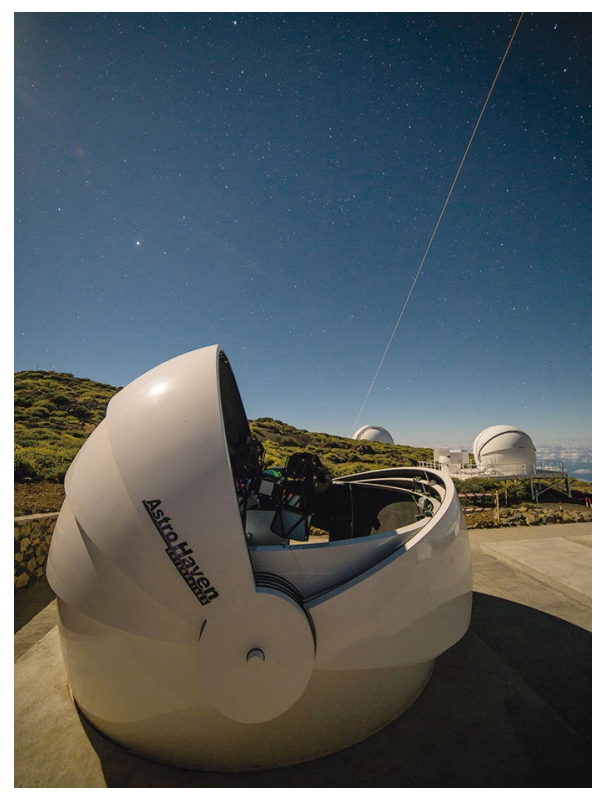

Fig. 1 | The GOTO project is an array of telescopes on custom mounts inside clamshell domes at the Roque de los Muchachos Observatory in La Palma, Spain. It aims to find visible counterparts to gravitational wavegenerating merger events.

Credit: K. Ulaczyk/University of Warwick

analysis strategy towards its principal goal, but at the same time is looking forward to a broad range of interesting time-domain science that can be pursued with GOTO.

GOTO is operated at the La Palma observing facilities of the University of Warwick on behalf of a consortium including the University of Warwick, Monash University, Armagh Observatory, the University of Leicester, the University of Sheffield, the National Astronomical Research Institute of Thailand (NARIT) and the Instituto de Astrofísica de Canarias (IAC).

\section{Danny Steeghs}

Danny Steeghs is in the Astronomy and Astrophysics Group at the University of Warwick, Coventry, UK. e-mail:D.T.H.Steeghs@warwick.ac.uk

Published online: 2 November 2017 https://doi.org/10.1038/s41550-017-0317-8 